\title{
Anisotropic viscoelastic models in large deformation for architectured membranes
}

\author{
Marie Rebouah $^{1,2}$ • Gregory Chagnon ${ }^{1,2}$ - Patrick Heuillet $^{3}$
}

\begin{abstract}
Due to the industrial elaboration process, membranes can have an in-plane anisotropic mechanical behaviour. In this paper, anisotropic membranes elaborated with two different materials were developed either by calendering or by inducing a force in one direction during the process. Experimental tests are developed to measure the differences of mechanical behaviour for both materials in different in-plane properties: stiffness, viscoelasticity and stress-softening. A uniaxial formulation is developed, and a homogenisation by means of a sphere unit approach is used to propose a three-dimensional formulation to represent the materials behaviour. An evolution of the mechanical parameters, depending on the direction, is imposed to reproduce the anisotropic behaviour of the materials. Comparison with experimental data highlights very promising results.
\end{abstract}

Keywords Viscoelasticity $\cdot$ Sphere unit model $\cdot$ Anisotropy $\cdot$ Stress-softening

\section{Introduction}

Depending on the process, rubber-like materials can be considered as initially isotropic or anisotropic. Even if isotropic behaviour is the most widespread, calender processing (Itskov and Aksel 2004; Diani et al. 2004; Caro-Bretelle et al. 2013) generates anisotropy by creating a privileged direction in the material. The differences of mechanical properties affect the stiffness, stress softening or viscoelastic properties. Even if rubber-like materials are isotropic, some induced anisotropy can be generated by the Mullins effect (Diani et al. 2009; Rebouah and Chagnon 2014b) for most materials.

Numerous studies have dealt with the modelling of rubber-like materials. In large deformations, the viscoelasticity is tackled either by the Boltzmann superposition principle

\footnotetext{
$凶$ G. Chagnon

gregory.chagnon@imag.fr

1 Universite Grenoble Alpes, TIMC-IMAG, 38000 Grenoble, France

2 CNRS, TIMC-IMAG, 38000 Grenoble, France

3 LRCCP, 60 Rue auber, Vitry sur Seine, France
} 
(Green and Rivlin 1957; Coleman and Noll 1963) which leads to the K-BKZ models or by internal variable models (Green and Tobolsky 1946). Many constitutive equations were proposed to describe different viscoelastic behaviours.

The modelling of calendered rubber sheet necessitates taking into account the initial anisotropy. Many constitutive equations are developed to describe anisotropic hyperelastic behaviour (Chagnon et al. 2015), these equations were often initially developed to describe soft biological tissues. Different equations were also developed to describe viscoelasticity for orthotropic materials or materials having one of two reinforced directions (Holzapfel et al. 2002; Bischoff et al. 2004; Haslach 2005; Quaglini et al. 2004; Vassoler et al. 2012). These constitutive equations rely on the representation of the material by a matrix with different reinforced directions, inducing the anisotropy in the material. The viscoelastic constitutive equations are introduced in the fibre modelling and can also be introduced in the matrix modelling. In a different way, Flynn and Rubin (2012) developed a discrete fibre model with dissipation for biological tissues. The model relies on a structural icosahedral model with six discrete fibres.

These models do not correspond to calendered materials. A calendered material can be represented as a macromolecular network in which the repartition of macromolecules was not equiprobable in space. A way to treat this problem is to describe the material by a uniaxial constitutive equation integrated in space considering different orientations. Different formalisms were proposed (Verron 2015). For soft tissues and rubber-like materials, the formalism proposed by Bažant and Oh (1986) is the most widespread. It was used to describe the stress softening or the viscoelasticity. Miehe and Göktepe (2005) were the first to use this formalism to describe the viscoelasticity but in an isotropic framework. The same formalism was later used by Diani et al. (2006), introducing induced anisotropy by the stress softening, but the viscoelasticity remained isotropic. Moreover, Rey et al. (2014) used this formalism to describe hysteresis loops but also in an isotropic approach. In fact, the discretisation in privileged directions is often used to induce anisotropy for hyperelasticity and stress softening, but not for viscoelasticity.

In this paper, we propose to characterise the mechanical behaviour of initially anisotropic rubber-like materials. The experimental data will be used to adapt the formalism proposed by Rebouah and Chagnon (2014a) to describe the anisotropic viscoelasticity and stress softening of the material.

Two rubber-like materials that possess an initial anisotropic behaviour are studied: first, a room temperature vulcanized silicone rubber which was made anisotropic by a stretching during reticulation, and second, a thermoplastic elastomer made anisotropic by the industrial process. In Part 2, the cyclic mechanical behaviour of the materials is described by means of a tensile test performed on specimens oriented in different directions in the plane of the membrane. In Part 3, the mono-dimensional constitutive equation is first described, and next the three-dimensional formulation is proposed. In Part 4, a discussion about the abilities of the constitutive equations to describe the two materials is proposed. Finally, a conclusion closes the paper.

\section{Experiments}

\subsection{Materials and specimen geometry}

In this paper, two materials that possess an anisotropic mechanical behaviour are used, a silicone rubber (RTV3428) and a thermoplastic elastomer (TPE). They are detailed in the next paragraphs. 


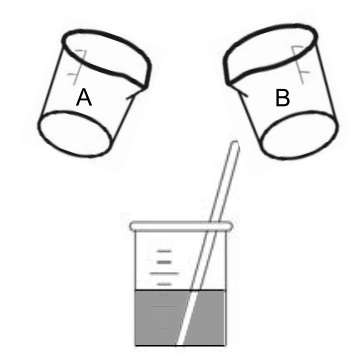

1. mixing two components

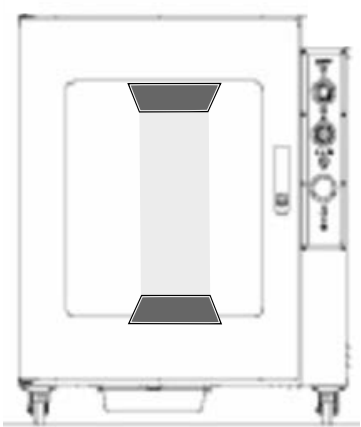

6. $2 \mathrm{~h}$ at $150^{\circ} \mathrm{C}$

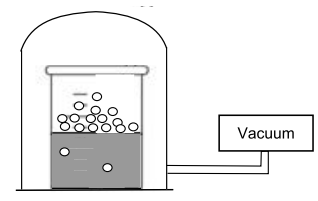

2. Vacuum
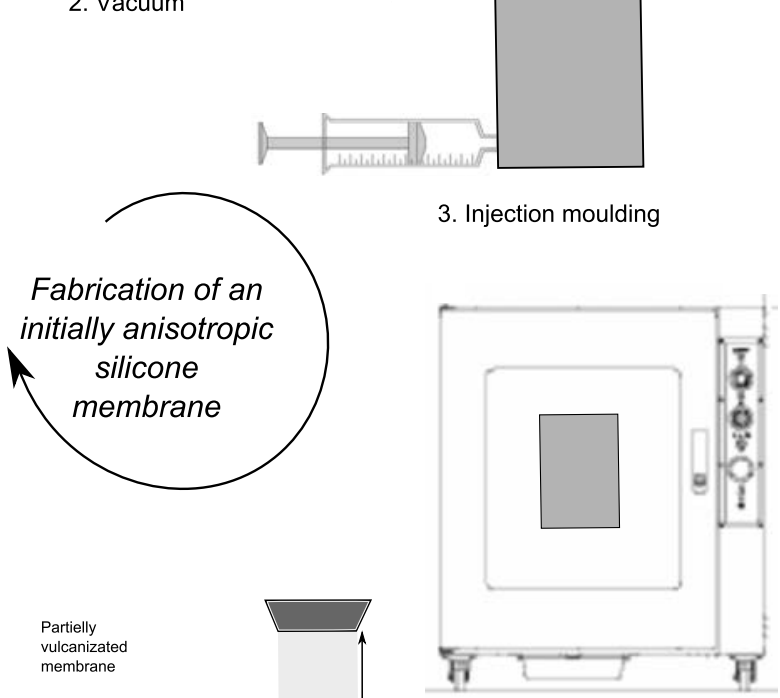

4. $22 \min$ at $70^{\circ} \mathrm{C}$

5. Applied a deformation to the membrane

Fig. 1 Elaboration of the microstructural architectured membrane of filled silicone

\subsubsection{RTV3428a}

An RTV3428 silicone rubber is used here, which was previously studied in other works (Rey et al. 2013). This material is initially isotropic and only has an anisotropy induced by Mullins effect (Machado et al. 2012). It is proposed to modify its microstructure by changing the elaboration process to generate an initially anisotropic behaviour. The process is illustrated in Fig. 1. To obtain this anisotropic plate, two components are mixed first. The mixture is then put into a vacuum pump and finally injected into a mould. The mould is put into the oven at $70^{\circ} \mathrm{C}$ for 22 minutes. The crosslinking of the obtained membrane is not fully performed after being removed from the mould. Next the membrane is installed in a clipping system made of two jaws and applying a constant displacement between the two extremities of the membrane (as represented in the fifth step in Fig. 1). The global deformation of the membrane in the system is about $60 \%$. The system is put into the oven at a temperature of $150{ }^{\circ} \mathrm{C}$ for two hours. The new obtained material is named RTV3428a. This process generates a preferential orientation of the macromolecular chains in the material. 

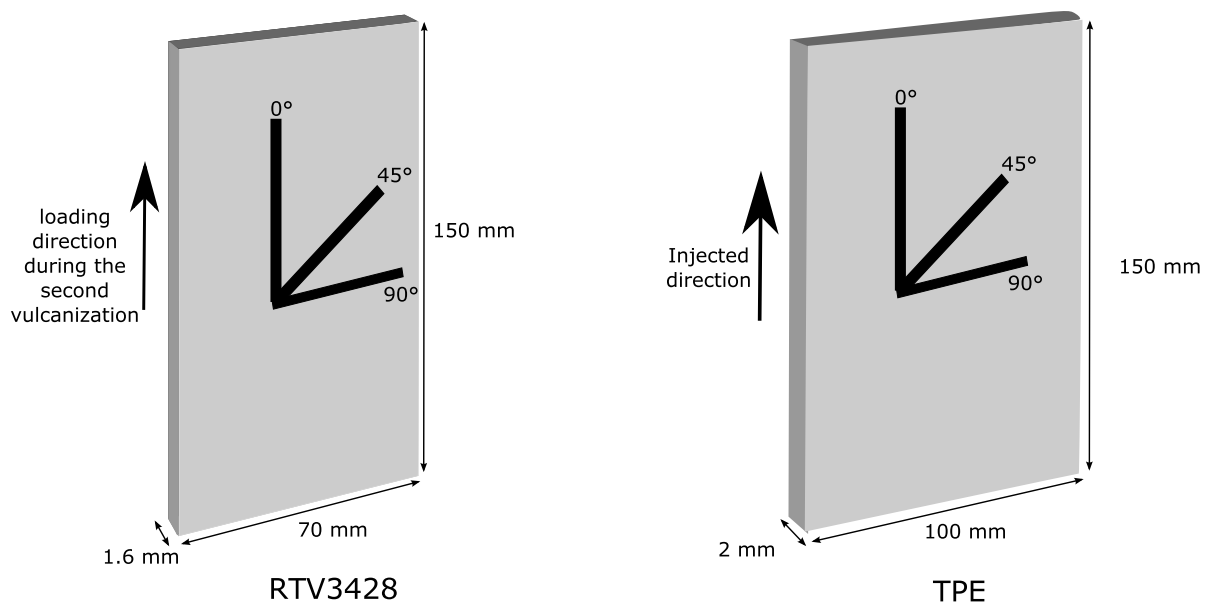

Fig. 2 Representation of the membrane and the samples oriented in different directions for the RTV3428a and the TPE materials

\subsubsection{TPE}

Different processes can be used to manufacture TPE (Caro-Bretelle et al. 2013). In this study, an industrial material provided by Laboratoire de recherches et de contrôle du caoutchouc et des plastiques (LRCCP) is used. This material is obtained by means of an injection process which gives it a predominant direction and makes it initially anisotropic.

\subsubsection{Specimen geometry}

Each material is initially elaborated as a membrane as illustrated in Fig. 2. The RTV3428a membrane dimensions after the second vulcanization are $150 \mathrm{~mm}$ in length, $70 \mathrm{~mm}$ in width and $1.6 \mathrm{~mm}$ in thickness; for the TPE the membrane dimensions are $150 \mathrm{~mm}$ in length, $100 \mathrm{~mm}$ in width and $2 \mathrm{~mm}$ in thickness. For each material tensile test samples $(20 \mathrm{~mm}$ long and $2 \mathrm{~mm}$ wide) are cut in the middle of the membrane to avoid edge effects. These specimens are cut with different angles $0^{\circ}, 45^{\circ}$ and $90^{\circ}$ compared to the preferential direction of the material, considering that $0^{\circ}$ matches the preferential direction imposed to the macromolecular chains for both processes as illustrated in Fig. 2.

\subsection{Loading conditions}

Mechanical tests were realised with a Gabo Eplexor $1500 \mathrm{~N}$ mechanical test machine with a load cell of $50 \mathrm{~N}$. Samples were submitted to a cyclic loading, two cycles up to a stretch $\lambda=1.5$, two cycles up to $\lambda=2$, and finally two cycles up to $\lambda=2.5$. The tests were carried out at a strain rate of $0.016 \mathrm{~s}^{-1}$. The loading history is detailed at the top of the Fig. 3 and Fig. 4.

\subsection{Results}

Figure 3 presents the results of the test for the three samples cut from the RTV3428a. The three samples do not have the same mechanical behaviour, and several phenomena 
Fig. 3 Cyclic tensile test A performed on the RTV3428a architectured membrane

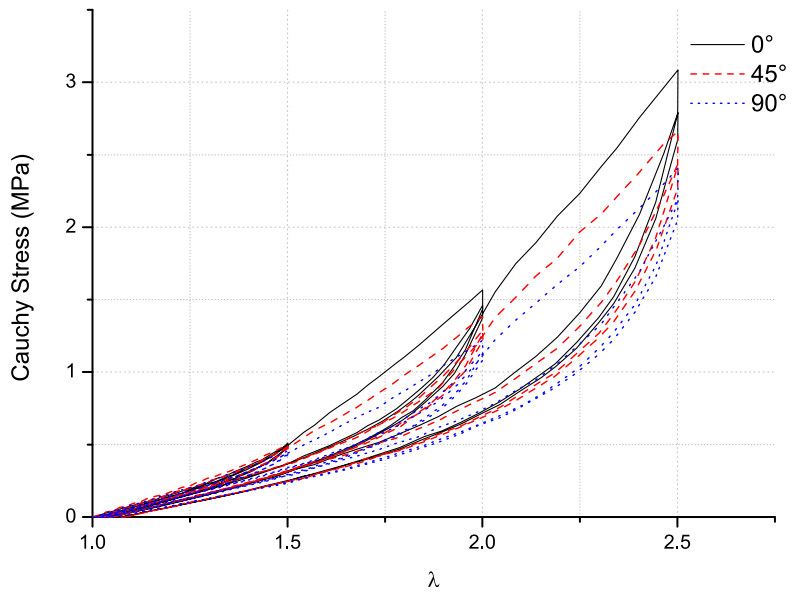

Fig. 4 Cyclic tensile test A performed on the microarchitectured membrane of TPE

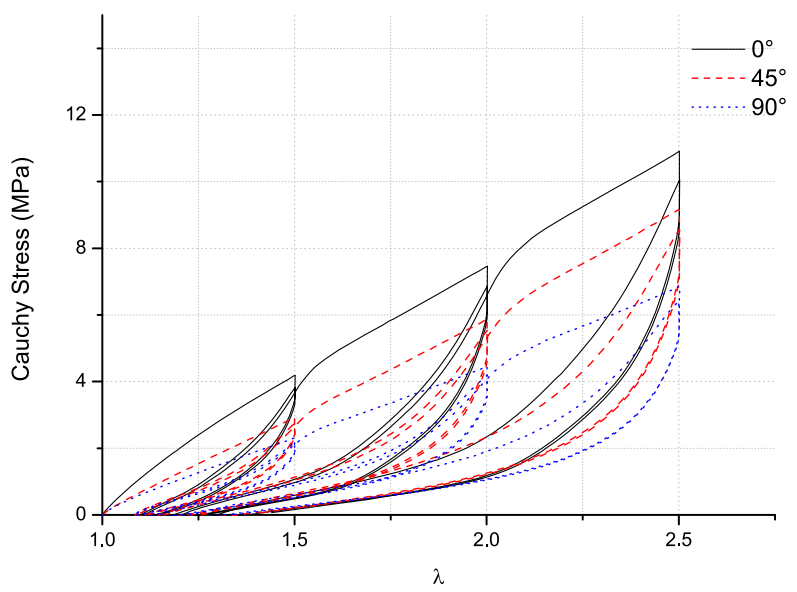

are observed. First, to evaluate the amount of anisotropy, an anisotropic factor $\xi$ is defined as the ratio of stresses for a stretch $\lambda=2.5$ for different orientations as $\xi=\sigma^{0^{\circ}}$ $(\lambda=2.5) / \sigma^{90^{\circ}}(\lambda=2.5)$. It permits qualitatively quantifying the anisotropy of the two materials. For the RTV3428a an anisotropic factor of approximately 1.3 can be calculated between the sample cut at $0^{\circ}$ and the one cut at $90^{\circ}$. This emphasises that the second vulcanization undergone by the membrane modifies the microstructure of this filled silicone and is efficient to generate anisotropy in the silicone rubber. The sample cut at $0^{\circ}$ (which is the same direction as the loading direction imposed during the second vulcanization) has the most important stress hardening compared to $45^{\circ}$ and $90^{\circ}$, the latter being the softest specimen. This test also highlights that the material has few viscous effects and permanent set even at slow strain rate. Stress softening is still the major non-linear effect associated with the mechanical behaviour.

Figure 4 presents the results for the same test obtained for the three samples of the TPE material. As before the anisotropic factor $\xi$ can be evaluated and is approximately equal to 1.5. As for the RTV3428a, stress softening, hysteretic behaviour and permanent set are also observed. It is to note that the stress softening and permanent set are very large. The 
Fig. 5 Definition of the configurations and of the rheological modelling
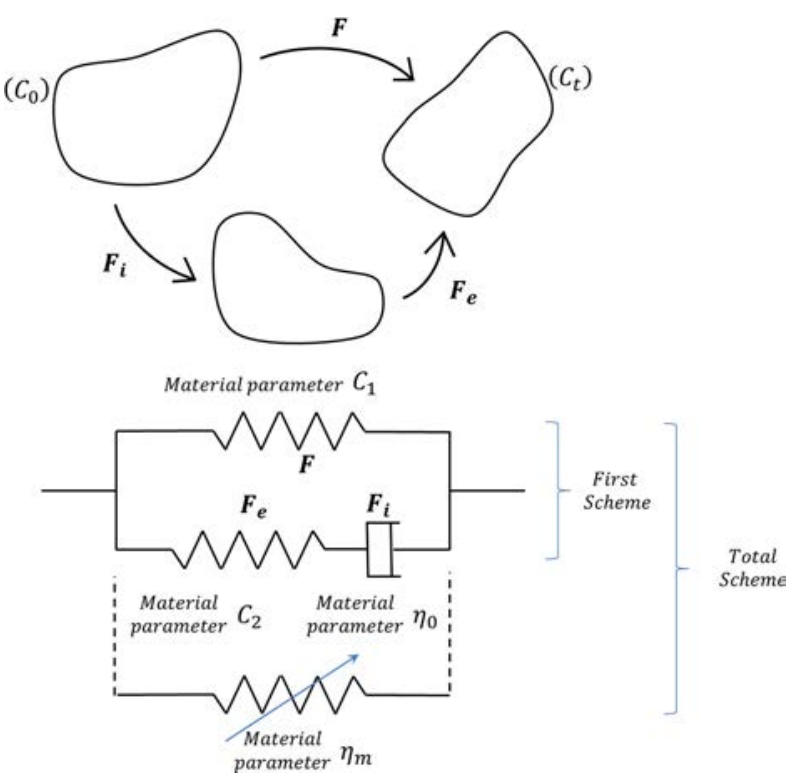

permanent set is approximately $20 \%$ of the maximum reached deformation. The viscoelastic effects are also very important as emphasised by the large hysteresis loops.

The observed phenomena are the same for the two materials. They both have an anisotropic mechanical behaviour with viscoelasticity, stress softening and permanent set for any loading direction. All the phenomena are even more amplified for the TPE than for the RTV3428a. The stress softening of the material between the first and the second cycles seems to be the same for any direction, this should mean that stress softening is not affected by the initial anisotropy. On the contrary, the stiffness and viscoelastic properties are modified for the two materials depending on the direction.

\section{Constitutive equations}

This section aims to detail the constitutive equation developed to describe the mechanical behaviour observed experimentally for both materials. This constitutive equation must take into account the anisotropy, stress softening and viscoelastic effects (including the permanent set) undergone by the materials. It is to note that the material is considered as a homogeneous structure and not as a matrix with reinforced fibres as it is classically done to represent the anisotropic materials (see, for example, Peña et al. 2011; Natali et al. 2009).

The constitutive equation relies on the representation of space by an integration of a uniaxial formulation by means of Bažant and Oh (1986) directions:

$$
\boldsymbol{\sigma}=\sum_{i}^{42} \omega^{(i)} \sigma^{(i)} \mathbf{a}_{n}^{(i)} \otimes \mathbf{a}_{n}^{(i)}
$$

where $\mathbf{a}_{n}^{(i)}$ are the normalized deformed directions, $\omega^{(i)}$ the weight of each direction and $\sigma^{(i)}$ the stress in the considered direction. The directions are represented in Fig. 6. The idea of the 

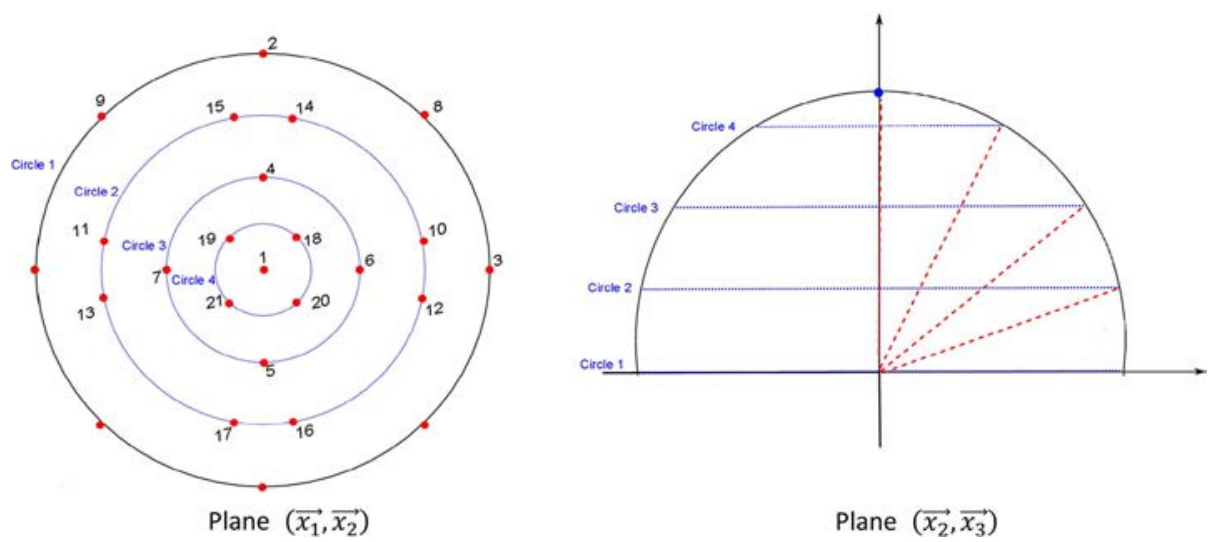

Fig. 6 Representation of a microsphere with a spatial repartition of $2 \times 21$ directions proposed by Bažant and $\mathrm{Oh}(1986)$

modelling is to propose the constitutive equation in tension-compression for each direction, i.e. $\sigma^{(i)}$.

A classical rheological scheme is used to model viscoelasticity; the first schema is illustrated in Fig. 5. The deformation gradient $\mathbf{F}$ is decomposed into an elastic part $\mathbf{F}_{\mathbf{e}}$ and an inelastic part $\mathbf{F}_{\mathbf{i}}$ between the initial configuration $\left(C_{0}\right)$ and the instantaneous configuration $\left(C_{t}\right)$. The formalism proposed by Huber and Tsakmakis (2000) is used. An application of the Second Principle of Thermodynamics leads to the equation of dissipation $D_{\text {int }}$, namely

$$
D_{\text {int }}=\boldsymbol{\sigma}: \mathbf{D}-\dot{W} \geq 0
$$

where $W$ is the strain energy (it can be decomposed into two parts, $W_{1}$ for the elastic branch of the model and $W_{2}$ for the inelastic branch of the model), $\sigma$ is the Cauchy stress tensor and D is the rate of deformation tensor. Huber and Tsakmakis (2000) proved that the sufficient condition to verify is

$$
2 \mathbf{F}_{e} \frac{\partial W}{\partial \mathbf{B}_{e}} \mathbf{F}_{e}: \mathbf{D}_{i} \geq 0
$$

The indices $e$ and $i$ are referring to the elastic and the inelastic parts of the model. As Huber and Tsakmakis (2000) detailed the simplest sufficient condition to satisfy, this equation is chosen as:

$$
\dot{\mathbf{B}}_{e}=\mathbf{L} \mathbf{B}_{e}+\mathbf{B}_{e} \mathbf{L}^{T}-\frac{2}{\eta_{0}} \mathbf{B}_{e}\left(\frac{\partial W_{2}}{\partial \mathbf{B}_{e}} \mathbf{B}_{e}\right)^{D}
$$

where $\mathbf{B}$ is the left Cauchy-Green tensor, $\mathbf{L}$ is the velocity gradient equal to $\mathbf{\mathbf { F }} \mathbf{F}^{-1}$ and ${ }^{D}$ strands for the deviatoric part of the tensor. Any constitutive equation can be used by assuming that each spring of the model is modelled by a neo-Hookean model (Treloar 1943), $C_{1}^{(i)}$ and $C_{2}^{(i)}$ are the material parameters of each branch. Index $i$ denotes that this model will be used in every directions of the microsphere decomposition. To be used, the governing equation must be written in uniaxial extension as

$$
\dot{\lambda}_{e}^{(i)}=\lambda_{e}{ }^{(i)} \frac{\dot{\lambda}^{(i)}}{\lambda^{(i)}}-4 \frac{C_{2}^{(i)}}{3 \eta_{0}^{(i)}}\left(\lambda_{e}^{(i)^{3}}-1\right)
$$


where $\lambda^{(i)}$ and $\lambda_{e}^{(i)}$ are the stretch and the elastic part of the stretch in the considered direction, and $\eta_{0}^{(i)}$ is a material parameter associated to each direction.

To take into account the stress softening phenomenon, a non-linear spring is added to the previous rheological scheme. It consists of a non-linear spring that can be added to the rheological scheme as illustrated in Fig. 6. Rebouah et al. (2013) proposed using an evolution function $\mathcal{F}^{(i)}$ that records the loading history of each direction, this function alters the stiffness of the non-linear spring:

$$
\mathcal{F}^{(i)}=1-\eta_{m}^{(i)} \sqrt{\frac{I_{1 \max }-I_{1}}{I_{1 \max }-3}}\left(\frac{I_{4 \max }^{(i)}-I_{4}^{(i)}}{I_{4 \max }^{(i)}-1}\right)\left(\frac{I_{4 \max }^{(i)}}{I_{4 \max }}\right)^{4}
$$

where $\eta_{m}^{(i)}$ is a material parameter, $I_{1}$ is the first invariant of Cauchy-Green tensor and $I_{4}^{(i)}$ is the fourth invariant associated to direction $i$. The term $I_{4 \max }^{(i)}$ is the maximum value reached at the current time for each direction, and $I_{4 \max }$ is the maximum value of $I_{4}$ for every direction.

Summing the viscoelastic part and the stress softening enables us to define the stress in direction $i$ considering that each direction endures only tension-compression, an incompressibility hypothesis is used to write the stress in each direction as

$$
\sigma^{(i)}=2 C_{1}^{(i)}\left(\lambda^{(i)^{2}}-\frac{1}{\lambda^{(i)}}\right)+2 C_{2}^{(i)}\left(\lambda_{e}^{(i)^{2}}-\frac{1}{\lambda_{e}^{(i)}}\right)+2 \lambda^{(i)^{2}} \mathcal{F}^{(i)}{\frac{\partial W_{c f}}{\partial I_{4}^{(i)}}}^{(i)}
$$

where $W_{c f}^{(i)}$ is the strain energy of the material oriented in direction $i$ for the stress softening part. Due to the differences observed experimentally between both materials, the strain energy $W_{c f}^{(i)}$ used to describe the RTV3428a and the TPE is different:

- The isotropic RTV3428 silicone rubber was already studied by Rebouah et al. (2013), and the same strain energy function was used in that study, namely

$$
\mathcal{W}_{c f}^{(i)}=K^{(i)}\left(I_{4}^{(i)}-1\right)^{2}
$$

where $K^{(i)}$ is a material parameter.

- The TPE has a very smooth behaviour with no strain hardening, as a consequence a square root function is chosen (Rebouah and Chagnon 2014b):

$$
\mathcal{W}_{c f}^{(i)}=\frac{K^{(i)}}{2} \int \sqrt{\frac{I_{4}^{(i)}-1}{I_{4}^{(i)}}} d I_{4}^{(i)}
$$

where $K^{(i)}$ is a material parameter.

The global dissipation of the model is obtained by summing the dissipation of each direction. As each dissipation is positive by construction of the evolution equation, the global dissipation is also positive.

To conclude, five parameters in each direction are used to handle the anisotropy of the material, $C_{1}{ }^{(i)}$ for the hyperelastic part, $C_{2}{ }^{(i)}$ and $\eta_{0}^{(i)}$ for the viscoelastic part and $K^{(i)}$ and $\eta_{m}^{(i)}$ for the stress softening part. The model needs the integration of a differential equation (Eq. (5)). An implicit algorithm is used to determine the equilibrium solution in each direction. 


\section{Comparison with experimental data}

\subsection{Parameter identification strategy}

The model has five material parameters in each direction. It is important not to fit globally all the material parameters, but to impose some restrictions due to experimental observations:

- First, the stiffness of the material is different depending on the direction $\left(0^{\circ}, 45^{\circ}\right.$ and $\left.90^{\circ}\right)$, this stiffness is principally controlled by the parameters controlling the hyperelasticity of the constitutive equation, $C_{1}{ }^{(i)}$ and $K^{(i)}$, these parameters must be different in the different directions.

- Second, no significant difference was observed for the stress softening for the three orientations, i.e. the difference between the first and second loadings. Thus, the material parameter describing the stress softening $\eta_{m}^{(i)}$ is chosen to be independent of the direction.

- Third, as exposed by Petiteau et al. (2013) and Rebouah and Chagnon (2014b), the hysteresis loop size depends both on the elastic parameter, $C_{2}$, and the time parameter, $\eta_{0}$. As the hysteresis loops are very similar, but at different stress levels, the governing parameter is $C_{2}{ }^{(i)}$. As a consequence, it is chosen to impose the same $\eta_{0}$ in all the directions.

It was experimentally observed that the stress is maximal for the privileged direction of the fabrication process. The variation of the mechanical parameters according to the spatial repartition enables us to increase or decrease the initial anisotropy of the material. According to the representation of the spatial repartition of Bažant and Oh (1986) as illustrated in Fig. 6, the closest directions of the microsphere to the preferential direction induced by the process (i.e., direction 1 in Fig. 6) are the directions with the largest material parameter values and minimum for the orthogonal directions 2 and 3 (of the microsphere). The values of the parameters are the same for the directions which are symmetrical with respect to the privileged direction of the sphere unit (direction 1). The values for the intermediary directions of the microsphere are obtained according to their relative position compared to direction 1 . The material parameters are supposed to vary linearly between the two extrema. All these choices permit us to avoid non-physical responses of the model for other loading conditions.

\subsection{RTV3428a}

According to the limitations detailed in the previous paragraph, the material parameters are fitted for the three samples with different orientations in comparison of the principal direction. The material parameters which values are independent of the directions are $\eta_{m}=4$ and $\eta_{0}=200 \mathrm{MPa} \mathrm{s}^{-1}$. The values of the other parameters are listed in Table 1.

Figure 7 presents a comparison between the experimental and theoretical tests for the three samples of RTV3428a with different orientations. The stiffness of the material is well described for any direction. The viscoelastic effects are also well described for the three directions. A difference can be observed for the second loading curves at the maximum stretch $\lambda=2.5$. This error corresponds to the stress softening part of the model. This could be improved by modifying the form of Eq. (6) by imposing a more important loss of stiffness. Nevertheless, the model is able to globally describe the anisotropic mechanical behaviour of the RTV3428a material. 
Table 1 Material parameters for the RTV3428a and the TPE

\begin{tabular}{|c|c|c|c|c|c|c|}
\hline \multirow[t]{2}{*}{$\mathrm{n}^{\circ}$} & \multicolumn{3}{|l|}{ RTV } & \multicolumn{3}{|l|}{ TPE } \\
\hline & $\overline{C_{1}}{ }^{(i)} \mathrm{MPa}$ & $C_{2}{ }^{(i)} \mathrm{MPa}$ & $K^{(i)} \mathrm{MPa}$ & $\overline{C_{1}}{ }^{(i)} \mathrm{MPa}$ & $C_{2}{ }^{(i)} \mathrm{MPa}$ & $K^{(i)} \mathrm{MP}$ \\
\hline 1 & 0.190 & 0.350 & 0.360 & 1.20 & 1.900 & 4.80 \\
\hline 2 & 0.133 & 0.280 & 0.252 & 0.48 & 0.570 & 1.92 \\
\hline 3 & 0.133 & 0.280 & 0.252 & 0.48 & 0.570 & 1.92 \\
\hline 4 & 0.161 & 0.280 & 0.306 & 0.96 & 1.235 & 3.84 \\
\hline 5 & 0.161 & 0.280 & 0.306 & 0.96 & 1.235 & 3.84 \\
\hline 6 & 0.161 & 0.280 & 0.306 & 0.96 & 1.235 & 3.84 \\
\hline 7 & 0.161 & 0.280 & 0.306 & 0.96 & 1.235 & 3.84 \\
\hline 8 & 0.123 & 0.315 & 0.144 & 0.48 & 0.570 & 1.92 \\
\hline 9 & 0.123 & 0.315 & 0.144 & 0.48 & 0.570 & 1.92 \\
\hline 10 & 0.161 & 0.210 & 0.216 & 0.72 & 1.045 & 2.88 \\
\hline 11 & 0.161 & 0.210 & 0.216 & 0.72 & 1.045 & 2.88 \\
\hline 12 & 0.161 & 0.210 & 0.216 & 0.72 & 1.045 & 2.88 \\
\hline 13 & 0.161 & 0.210 & 0.216 & 0.72 & 1.045 & 2.88 \\
\hline 14 & 0.161 & 0.210 & 0.216 & 0.72 & 1.045 & 2.88 \\
\hline 15 & 0.161 & 0.210 & 0.216 & 0.72 & 1.045 & 2.88 \\
\hline 16 & 0.161 & 0.210 & 0.216 & 0.72 & 1.045 & 2.88 \\
\hline 17 & 0.161 & 0.210 & 0.216 & 0.72 & 1.045 & 2.88 \\
\hline 18 & 0.171 & 0.315 & 0.324 & 1.08 & 1.520 & 4.32 \\
\hline 19 & 0.171 & 0.315 & 0.324 & 1.08 & 1.520 & 4.32 \\
\hline 20 & 0.171 & 0.315 & 0.324 & 1.08 & 1.520 & 4.32 \\
\hline 21 & 0.171 & 0.315 & 0.324 & 1.08 & 1.520 & 4.32 \\
\hline
\end{tabular}

\subsection{TPE}

As before the material parameters of the TPE are fitted to the tensile tests of the three specimens with different orientations. The material parameters which values are independent of the directions are $\eta_{0}=500 \mathrm{MPas}^{-1}, \eta_{m}=8$. The values of the other parameters are listed in Table 1 and are obtained by the same strategy as the one described for the RTV3428a.

Figure 8 presents a comparison of the model with experimental data. The variations of stiffness of the material with the directions are well described. Nevertheless, the model is not able to describe very large hysteresis loops and very important stress softening. Important differences are observed for the model according to the direction, but the size of the hysteresis is underestimated. This is due to the form of the constitutive equations that were chosen. Only neo-Hookean constitutive equations were used in the viscoelastic part, and it is well known that this model cannot describe large variations. Moreover, the governing equation of the viscoelasticity (i.e., Eq. (5)) is a very simple equation that also cannot take into account large non-linearity of the mechanical behaviour.

Nevertheless, even if the proposed model is a first approach written with simple constitutive elements, all the phenomena are qualitatively described. The limits correspond to the limits of each part of the constitutive equation. 

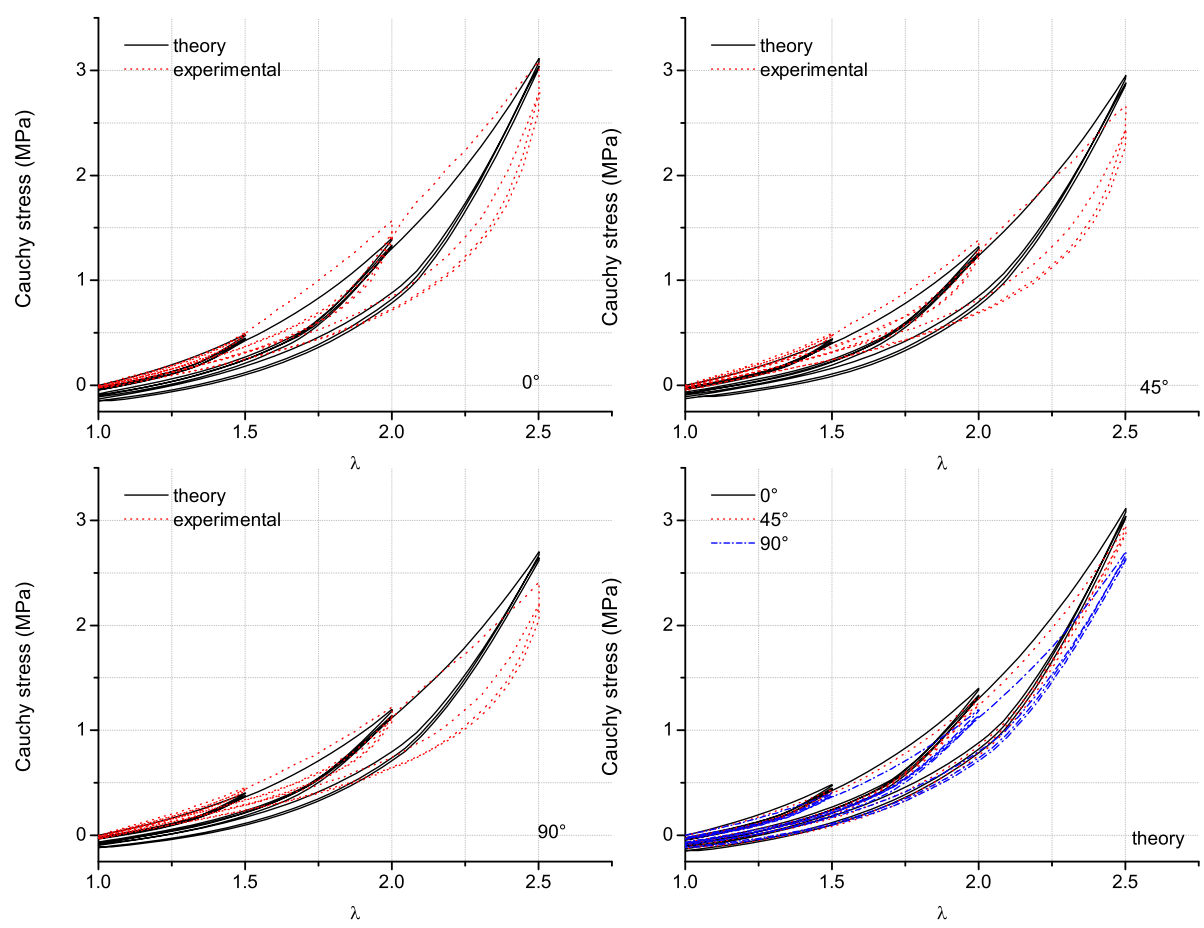

Fig. 7 Comparison with the experimental data of cyclic tensile test on RTV3428a for the different samples with different orientations

\subsection{Discussion}

The model succeeds in depicting the anisotropic viscoelasticity with stress softening mechanical behaviour of architectured membranes. The use of different material parameters in the different directions leads to an important number of parameters. A global fit of the parameters could lead to parameter values with no physical meaning. By analysing the experimental data, a strategy was proposed to fit the material parameters. As exposed in the Introduction, constitutive equations developed in the literature to model anisotropic viscoelasticity often rely on an isotropic matrix reinforced with some viscoelastic fibres. These models were principally elaborated for soft biological tissues and could be applied in a phenomenological approach to the two materials tested in this paper. It would consist in considering the rubber as a soft matrix having the mechanical properties of the soft direction, reinforced by fibres in the predominant direction of the material. Even if this approach were to succeed in describing the material, it would not characterise the macromolecular network of the material.

All the equations in the literature to model for viscoelasticity can be written in tensioncompression and introduced into the present model, by replacing Eq. (5). This would permit us to represent non-linear viscoelasticity. 

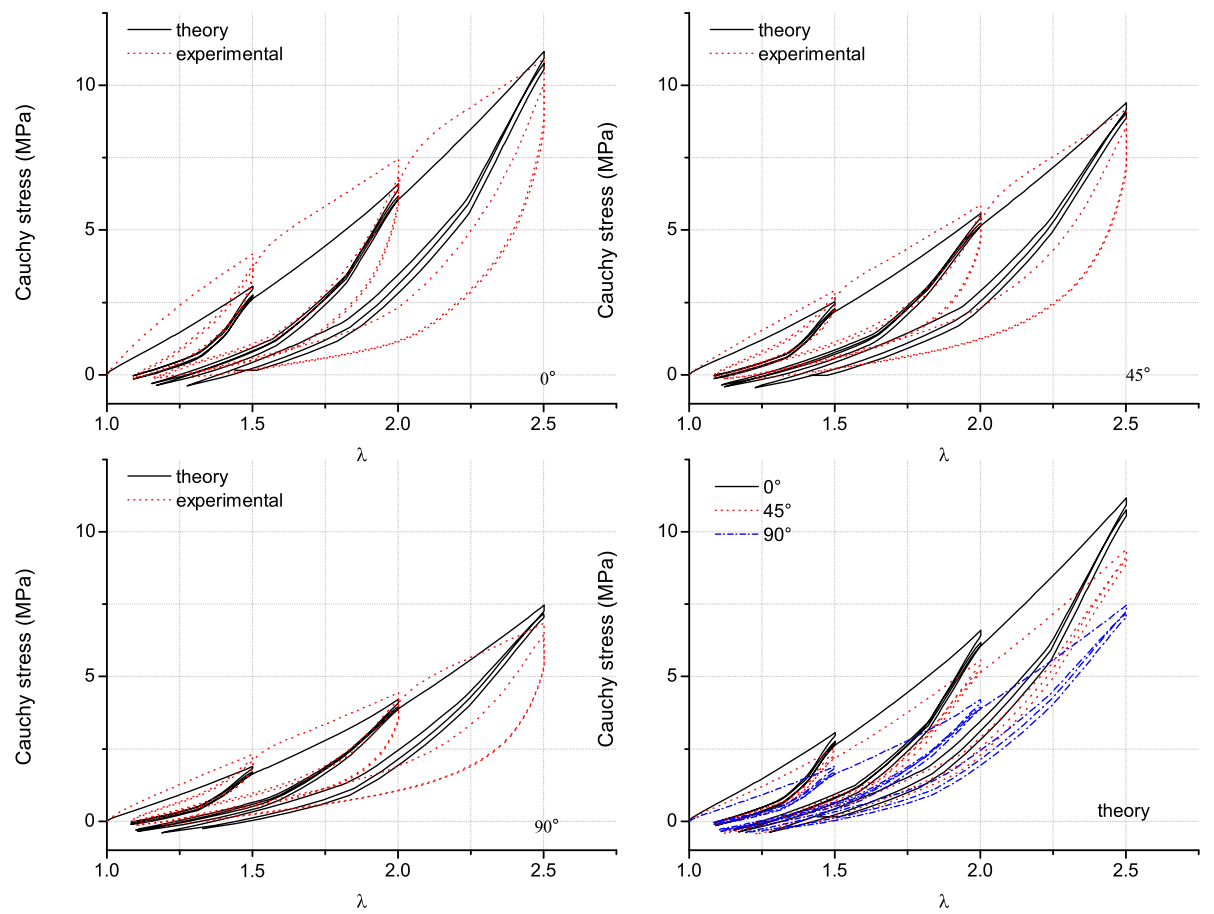

Fig. 8 Comparison with the experimental data of cyclic tensile test on TPE for the three samples with different orientations

\section{Conclusion}

This paper developed a study of anisotropic materials. Two micro-mechanical architectured materials were obtained in two different ways. A silicone rubber was turned anisotropic by applying a deformation state during the second reticulation, and an initially anisotropic injected TPE was used. In both cases an orientation of the macromolecular chains was imposed to the material to create a microstructural architecture. The anisotropic membranes were tested in their plane, highlighting their anisotropic mechanical behaviour.

A three-dimensional equation was obtained by considering the integration in space of a uniaxial equation with the 42 directions of Bažant and Oh (1986). This equation describes hyperelasticity, viscoelasticity and stress softening. In this first approach, we chose to use simple constitutive equations to prove the feasibility of the method. As a consequence, a neoHookean model was chosen to describe the elasticity and a simple linear equation for viscoelasticity. The anisotropy was obtained by considering that the material parameters could be different in all directions. Nevertheless, we chose to limit the variations of parameter values depending on the directions. This permitted us to limit the number of independent mechanical parameters. It was even possible to use different parameter values in every direction.

It appears that the model succeeded in qualitatively describing all the phenomena. When the phenomena (stress softening, hysteresis) were not too large, the model succeeded also in quantitatively describing the tests. Some errors between experimental data and the model appeared when the phenomena became too large. This was due to the use of simple elements for hyperelasticity and viscoelasticity. Indeed, the most robust constitutive equation 
for hyperelasticity, stress softening and viscoelasticity should be used when the phenomena are very large. For instance, the neo-Hookean hyperelastic equation should be replaced by a model accounting for stress hardening, or the viscoelastic equation should be replaced by a non-linear one as in, e.g. Bergstrom and Boyce (1998).

Acknowledgements This work is supported by the French National Research Agency Program ANR-12BS09-0008-01 SAMBA (Silicone Architectured Membranes for Biomedical Applications).

\section{References}

Bažant, Z.P., Oh, B.H.: Efficient numerical integration on the surface of a sphere. Z. Angew. Math. Mech. 66, 37-49 (1986)

Bergstrom, J.S., Boyce, M.C.: Constitutive modeling of the large strain time dependant behavior of elastomers. J. Mech. Phys. Solids 46(5), 931-954 (1998)

Bischoff, J.E., Arruda, E.M., Grosh, K.: A rheological network model for the continuum anisotropic and viscoelastic behavior of soft tissue. Biomech. Model. Mechanobiol. 3, 56-65 (2004)

Caro-Bretelle, A., Ienny, P., Leger, R.: Constitutive modeling of a SEBS cast-calender: large strain, compressibility and anisotropic damage induced by the process. Polymer 54, 4594-4603 (2013)

Chagnon, G.C., Rebouah, M., Favier, D.: Hyperelastic energy densities for soft biological tissues: a review. J. Elast. 120, 129-160 (2015)

Coleman, B.D., Noll, W.: Arch. Ration. Mech. Anal. 13, 167-178 (1963)

Diani, J., Brieu, M., Vacherand, J.M., Rezgui, A.: Directional model isotropic and anisotropic hyperelastic rubber-like materials. Mech. Mater. 36, 313-321 (2004)

Diani, J., Brieu, M., Gilormini, P.: Observation and modeling of the anisotropic visco-hyperelastic behavior of a rubberlike material. Int. J. Solids Struct. 43, 3044-3056 (2006)

Diani, J., Fayolle, B., Gilormini, P.: A review on the Mullins effect. Eur. Polym. J. 45, 601-612 (2009)

Flynn, C., Rubin, M.B.: An anisotropic discrete fibre model based on a generalised strain invariant with application to soft biological tissues. Int. J. Eng. Sci. 60, 66-76 (2012)

Green, A., Rivlin, R.: The mechanics of non-linear materials with memory: part I. Arch. Ration. Mech. Anal. 1, 1-21 (1957)

Green, M.S., Tobolsky, A.V.: A new approach for the theory of relaxing polymeric media. J. Chem. Phys. 14, 87-112 (1946)

Haslach, H.W.: Nonlinear viscoelastic, thermodynamically consistent, models for biological soft tissue. Biomech. Model. Mechanobiol. 3, 172-189 (2005)

Holzapfel, G.A., Gasser, T.C., Stadler, M.: A structural model for the viscoelastic behavior of arterial walls: continuum formulation and finite element analysis. Eur. J. Mech. A, Solids 21, 441-463 (2002)

Huber, A., Tsakmakis, C.: Finite deformation viscoelasticity laws. Mech. Mater. 32, 1-18 (2000)

Itskov, M., Aksel, N.: A class of orthotropic and transversely isotropic hyperelastic constitutive models based on a polyconvex strain energy function. Int. J. Solids Struct. 41, 3833-3848 (2004)

Machado, G., Chagnon, G., Favier, D.: Induced anisotropy by the Mullins effect in filled silicone rubber. Mech. Mater. 50, 70-80 (2012)

Miehe, C., Göktepe, S.: A micro-macro approach to rubber-like materials. Part ii: the micro-sphere model of finite rubber viscoelasticity. J. Mech. Phys. Solids 53, 2231-2258 (2005)

Natali, A.N., Carniel, E.L., Gregersen, H.: Biomechanical behaviour of oesophageal tissues: material and structural configuration, experimental data and constitutive analysis. Med. Eng. Phys. 31, 1056-1062 (2009)

Peña, E., Martins, P., Mascarenhasd, T., Natal Jorge, R.M., Ferreira, A., Doblaré, M., Calvo, B.: Mechanical characterization of the softening behavior of human vaginal tissue. J. Mech. Behav. Biomed. 4, 275-283 (2011)

Petiteau, J.-C., Verron, E., Othman, R., Sourne, H., Sigrist, J.-F., Barras, G.: Large strain rate-dependent response of elastomers at different strain rates: convolution integral vs. internal variable formulations. Mech. Time-Depend. Mater. 17, 349-367 (2013)

Quaglini, V., Vena, P., Contro, R.: A discrete-time approach to the formulation of constitutive models for viscoelastic soft tissues. Biomech. Model. Mechanobiol. 3, 85-97 (2004)

Rebouah, M., Chagnon, G.: Extension of classical viscoelastic models in large deformation to anisotropy and stress softening. Int. J. Non-Linear Mech. 61, 54-64 (2014a)

Rebouah, M., Chagnon, G.: Permanent set and stress softening constitutive equation applied to rubber like materials and soft tissues. Acta Mech. 225, 1685-1698 (2014b) 
Rebouah, M., Machado, G., Chagnon, G., Favier, D.: Anisotropic Mullins softening of a deformed silicone holey plate. Mech. Res. Commun. 49, 36-43 (2013)

Rey, T., Chagnon, G., Le Cam, J.-B., Favier, D.: Influence of the temperature on the mechanical behavior of two silicone rubbers above crystallization temperature. Polym. Test. 32, 492-501 (2013)

Rey, T., Chagnon, G., Favier, D., Le Cam, J.-B.: Hyperelasticity with rate-independent microsphere hysteresis model for rubberlike materials. Comput. Mater. Sci. 90, 89-98 (2014)

Treloar, L.R.G.: The elasticity of a network of long chain molecules (I and II). Trans. Faraday Soc. 39, 36-64 (1943); 241-246

Vassoler, J.M., Reips, L., Fancello, E.A.: A variational framework for fiber-reinforced viscoelastic soft tissues. Int. J. Numer. Methods Eng. 89, 1691-1706 (2012)

Verron, E.: Questioning numerical integration methods for microsphere (and microplane) constitutive equations. Mech. Mater. 89, 216-228 (2015) 